\title{
Correspondence
}

Correspondents should note that space is limited and shorter letters have a greater chance of publication. The Editors reserve the right to cut letters and also to eliminate multitudinous references. Please try to be concise, strictly relevant and interesting to the reader.

\section{THE CORPUS CALLOSUM AND BRAIN FUNCTION IN SCHIZOPHRENIA} DEAR SIR,

Jones and Miller (Journal, December, 1981, 139, 553-7) have added further support to the growing evidence that an impairment of corpus callosum transmission is one feature of the cerebral dysfunction accompanying schizophrenia. Their findings also support a model of brain organization in this disorder discussed by Shaw, Resek and Coulter 'EEG Coherence, Lateral Preference and Schizophrenia' paper submitted for publication.

These latter authors infer from their experimental EEG findings that brain organization in schizophrenia is diffuse like that found in healthy lefthanded individuals. There is evidence that such an organization requires more interhemisphere integration via the corpus callosum than the more common lateralized organization present in healthy right handers.

Jones and Miller interpret their finding of negligible ipsilateral/contralateral latency differences of the early somatosensory evoked response in schizophrenia as follows: First, that the corpus callosum is not conducting at all and second, that the ipsilateral response is produced by ipsilateral pathways from the brain stem. The latter is compatible with the suggestion of Shaw et al that brain organization in schizophrenia is like that in lefthanders. This follows from Kinsbourne's (1980) hypothesis that in the lefthander, the diffuse organization develops from bilateral cerebral activation during the adoption of a verbal mental set as a result of a "less laterally polarized brain stem (thalamic) influence that is projected to both cerebral hemispheres". Such organization may account for the sometimes reported, but tenuous, association between lefthanders and schizophrenia (Taylor et al, 1980).

Split-brain patients and healthy lefthanders do not show the behavioural disorder of schizophrenia. Jones and Miller's suggestion of bilateral brainstem influence is supported by Shaw et al's EEG evidence. It reinforces the latter's conclusion that impaired corpus callosum transmission, together with a brain organized to need it more than most, may contribute to the behavioural and intellectual disturbances of schizophrenia. It would be valuable to apply Jones and Miller's experiment to healthy lefthanders. The outcome may help to decide whether their result was dependent only on faulty corpus callosum transmission or due to the postulated bilateral brainstem projection.

It is of interest that Rosenthal and Bigelow's (1972) finding of an enlarged corpus callosum in schizophrenia has initiated so many studies showing impaired corpus callosum transmission in this disorder. These 1972 results were based on only 10 patients and do not appear to have been replicated.

MRC Clinical Psychiatry Unit,

John C. SHaW

Graylingwell Hospital,

Chichester, Sussex PO19 4PQ

\section{References}

KINSBOURNE, M. (1980) A model for the ontogeny of cerebral organisation in non-right-handers. In Neuropsychology of Left-handedness (ed. J. Herron). New York: Academic Press, pp 177-85.

Rosenthal, R. \& Bigelow, L. B. (1972) Quantitative brain measurements in chronic schizophrenia. British Journal of Psychiatry, 121, 259-64.

Taylor, P. J., Dalton, R. \& Fleminger, J. J. (1980) Handedness in schizophrenia. British Journal of Psychiatry, 136, 375-83.

\section{DeAR SiR,}

I believe the recent article by Jones and Miller (1981) entitled 'Functional Tests of the Corpus Callosum in Schizophrenia' deserves comment as it makes untenable assumptions, presents results indicating, amongst other things, neuropathology in the normal control sample, and reaches conclusions which are unwarranted.

Jones and Miller base much of their introductory opinions on unreplicated results (Beaumont and Dimond, 1973), conclusions since altered by the author (Flor-Henry, 1976) or on interpretation of results contradictory to the interpretation of the original authors (Gruzelier and Venables, 1974). While it is conceivable that there is a callosal transmission problem in schizophrenia it cannot be 
denied that the overwhelming evidence at the moment does not support the idea.

However, the truly worrying aspect of this paper is the results. In every respect, the morphology of the SEP in the normal controls differs substantially from results shown by Salamy (1978) whom the authors cite as the basis of their technique. The amplitudes (especially $\mathrm{N}_{1}, \mathrm{P}_{2}$ ) in Jones and Miller's normals are less than half the size shown by Salamy (1978). In fact the waveforms shown by Jones and Miller are sufficiently unlike Salamy's (1978) as to lead one to believe that they simply recorded their SEPs from sites other than those stated (e.g. $1-2 \mathrm{~cm}$ anterior or posterior to the point $6-7 \mathrm{~cm}$ lateral to the midline in the internal plane). However, even at such sites the waveform remains fairly intact and more importantly the peak latencies at such sites approximate those seen at sites $3 \mathrm{~cm}$ posterior to the lateral point. It is this aspect of the Jones and Miller results which is so confusing. The latencies of $P_{1}, N_{1}$ and $P_{2}$ in Jones and Miller are surprising. In the contralateral condition all three peaks occurred before $50 \mathrm{msec}$ whereas in the ipsilateral condition all other peaks occurred after $50 \mathrm{msec}$ (their Fig 1). This is in contrast to Salamy who observed that generally only the $P_{1}$ peak occurred before $50 \mathrm{msec}$.

The next issue concerns the gross discrepancy between Jones and Miller and Salamy (1978) on the ipsilateral-contralateral mean latency differences for the three peaks. The differences reported by Salamy (1978) range from approximately $5 \mathrm{msec}$ to at the most $10 \mathrm{msec}$ in his adult sample. Jones and Miller present differences ranging from approximately $8 \mathrm{msec}$ to $26 \mathrm{msec}$. The latter latency is greater than Salamy observed in $4 \frac{1}{2}$-year-old subjects. In fact, it is likely that in statistical terms, the schizophrenic patients not the controls of Jones and Miller more closely approximate Salamy's (1978) normal adult controls. Further this finding must have been common given the results shown in their Fig 2. Even this proposal must be treated cautiously insomuch as out of 36 ipsilateralcontralateral latency differences reported for patients fully 19 of them reflected earlier ipsilatreal latencies. This result receives no mention by Jones and Miller. Considering the impressive array of evidence reported and cited by Salamy (1978) that ipsilateral responses are slower than contralateral responses almost certainly due to being transmitted commissurally it is surprising that Jones and Miller offered no explanation for their ability to record a SEP at a secondary site before it had reached the primary location.

The suggestion of Jones and Miller that the corpus callosum of schizophrenics "is not conducting at all" is extremely unlikely. If this were so, results obtained by
Sperry and his colleagues with split-brain patients would be easily demonstrable in schizophrenic patients-a proposition well known to be not the case. As I mentioned earlier, transmission problems are a possibility but a total lack of function is simply not tenable.

Finally, it should be noted that Salamy's (1978) technique is likely to be assessing only the large diameter $(2.5 \mu \mathrm{m})$ myelinated axons which represent 10 per cent of myelinated callosal axons. Further, something in the region of 40 per cent of human callosal axons are unmyelinated (Swadlow et al, 1979). The end result of all this is that even if the results of Jones and Miller were not so unlikely, reaching conclusions such as total callosal block based on less than 5 per cent of callosal axons would be inadvisable.

Jones and Miller observe in the first paragraph of their Discussion the possible reasons for their unusual results. Whether their explanations are valid or not is open to question, but one is compelled to suggest that given their exceptional results it might have been worthwhile ascertaining just what they were measuring and considering the issues rather more thoroughly.

Wellcome Research Fellow, JOHN F. CONNOLLY

Department of Psychiatry, Charing Cross Hospital Medical School, London

\section{References}

Beaumont, J. G. \& Dimond, S. J. (1973) Brain disconnection and schizophrenia. British Journal of Psychiatry, 123, 661-2.

FloR-HeNRY, P. (1976) Lateralized temporal-limbic dysfunction and psychopathology. Annals of the New York Academy of Science, 280, 777-95.

Gruzelier, J. H. \& Venables, P. H. (1974) Bimodality and lateral asymmetry of skin conductance orienting activity in schizophrenics: replication and evidence of lateral asymmetry in patients with depression and disorders of personality. Biological Psychiatry, 8, 55-73.

JoNes, G. H. \& MilleR, J. J. (1981) Functional tests of the corpus callosum in schizophrenia. British Journal of Psychiatry, 139, 553-7.

Salamy, A. (1978) Commissional transmission: maturational changes in humans. Science, 200, 1409-11.

Swadlow, H. A., Geschwind, N. \& Waxman, S. O. (1979) Commissural transmission in humans. Science, 204, $530-1$.

DEAR SIR,

Following the suggestion that the corpus callosum be examined carefully in schizophrenia (Journal, December, p 556) we have been able to do so in two instances.

A 53-year-old man drowned after a 21 year history of schizophrenia, with clear passivity phenomena and 